\title{
Utilizing optical coherence tomography in the diagnosis and management of cystoid macular edema with topical treatment
}

This article was published in the following Dove Press journal:

Clinical Optometry

18 November 2010

Number of times this article has been viewed

\section{Michael Dobos}

Canton Veterans Affairs Community based Outpatient Clinic, Canton, $\mathrm{OH}$, USA
Correspondence: Michael Dobos

Canton VA CBOC, 733 Market Ave South,

Canton, OH 44702, USA

Email dobos.10@osu.edu

\begin{abstract}
Cystoid macular edema (CME) is characterized by inflammation at the fovea caused by the breakdown of the blood-retinal barrier, resulting in the accumulation of fluid-filled cysts in the outer plexiform and inner nuclear layers of the retina. Physiologically, CME is caused by prostaglandin release, resulting in retinal vasodilation and vasopermeability. The etiology of CME is most often due to a history of ocular surgery or inflammation. Historically, fluorescein angiography has been the main diagnostic test for CME with its distinguishing "flower-petal" pattern. In this case report, optical coherence tomography (OCT) is utilized in the management of CME treated with ocular medication.
\end{abstract}

Keywords: cystoid macular edema, CME, optical coherence tomography

\section{Introduction}

Cystoid macular edema (CME) is characterized by inflammation at the fovea caused by the breakdown of the blood-retinal barrier, resulting in the accumulation of fluidfilled cysts in the outer plexiform and inner nuclear layers of the retina. Physiologically, CME is caused by prostaglandin release, resulting in retinal vasodilation and vasopermeability. The etiology of CME is most often due to a history of ocular surgery or inflammation. Other causes of CME include diabetic retinopathy, retinal vein occlusions, and uveitis. ${ }^{1-4}$ Fluorescein angiography (FA) has been the main diagnostic test for CME. The accumulation of fluorescein dye in the outer plexiform layer of the retina, with its radial arrangement of fiber, leads to the distinguishing "flower-petal" pattern. In this case report, optical coherence tomography (OCT) is utilized in the management of CME treated with ocular medication.

OCT provides accurate and repeatable cross-sectional and quantitative measures of macular edema of various causes. ${ }^{5,6}$ The clinical use of OCT can help analyze macular conditions in conjunction with FA or without FA. ${ }^{5}$ The OCT scans in this case represent a useful way of monitoring the treatment of this case's CME with ocular medication.

\section{Case report}

A 51-year-old white male presented for the first time to the Chillicothe Veterans Affairs Medical Center Eye Clinic with decreased vision and pain oculus sinister (OS) on July 22, 2005. The patient had noticed redness and photophobia OS. He reported these signs and symptoms had started on the previous day. The patient reported that his last eye examination was two to three years earlier when he was just checked for glasses although not given a dilated fundus exam. The patient's past ocular history included a history of two vitrectomies OS in 1999 due to an intraocular metal foreign body. 
The patient reported that he had also had laser retinopexy OS to repair a retinal detachment subsequent to going back to work too early after the second vitrectomy. The patient's medical history was positive for hypertension and for a mini-stroke a few years earlier. He was oriented to time, place, and person.

Best-corrected visual acuity (VA) was measured to be 20/20 oculus dexter (OD) and 20/50- OS at distance. Upon observation of the patient during VA testing, the author questioned the patient who replied that he had "to look around the center of his vision" OS. Amsler grid testing was normal OD; however, a small area of central metamorphopsia was noted OS. Pupils were equally round and reactive to light, and no afferent pupil defect was noted oculus uterque (OU). Confrontation fields were full to finger counting OU. Extraocular muscles were unrestricted in all gazes. Intraocular pressures (IOPs) were $15 \mathrm{mmHg}$ OD and OS with applanation tonometry.

Anterior segment evaluation by slit-lamp examination revealed quiet eyelids and eyelashes OU. Bulbar conjunctiva was normal OD; however, grade 2+ diffuse injection was seen OS. Scarring was also seen superiorly OS due to the previous intraocular foreign body. Cornea OD was clear and quiet. Fine keratic precipitates were observed on the inferior corneal endothelium OS. Anterior chamber angles were deep and quiet OD and deep with $1+$ cell and flare OS. The iris was within normal limits OD, although atrophy was seen superiorly OS due to the history of the intraocular foreign body.

The patient was dilated using one drop 1\% Mydriacyl ${ }^{\circledR}$ and one drop $2.5 \%$ phenylephrine OU. Once the patient was fully dilated, an evaluation of the posterior segment by slit lamp with a 90D fundus lens and by binocular indirect opthalmoloscope revealed mild aging changes to the lens OD (1+ nuclear sclerotic cataract OD) and a posterior chamber intraocular lens OS (well centered, posterior capsule intact with no fibrosis). Vitreous floaters were notes OU, no pigment/cells were seen OU.

Fundus assessment revealed normal optic nerves with a cup-to-disc ratio of $0.3 / 0.3 \mathrm{OU}$. The neuroretinal rims were healthy and intact and the nerves showed normal color and margins OU. Maculae examination showed normal findings OD; however, a hazy, elevated macula was seen OS (Figure 1).

Macular thickening was also appreciated OS. Possible cystoid spots were also seen within the elevated area. Contact lens fundoscopy OS revealed small fluid-filled cysts in the area of thickening. Blood vessels shaved normal course and caliber OU. Peripheral retina was attached OD and

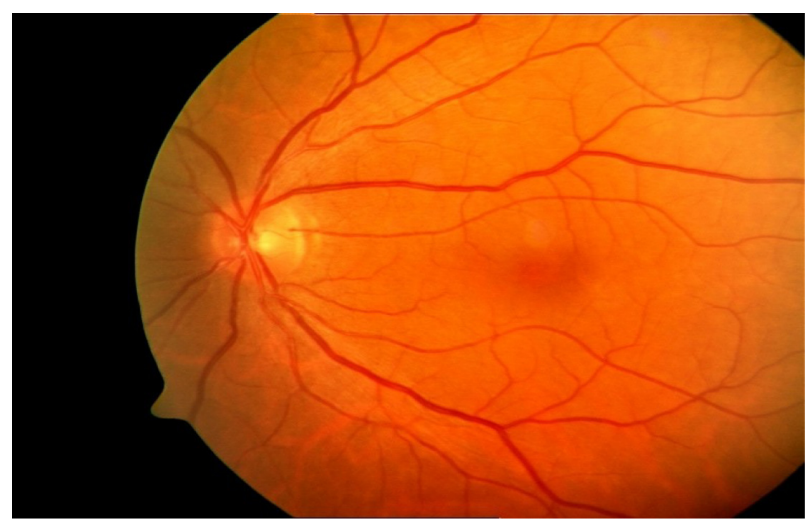

Figure I Fundus photograph OS.

Abbreviation: OS, oculus sinister.

attached OS with chorioretinal scarring status post laser retinopexy. OCT macula scans were performed OU and yielded foveal thickness results of $270 \mu \mathrm{m}$ OD and $572 \mu \mathrm{m}$ OS (see Figures 2 and 3).

The differential diagnoses considered in this case included stage 1 macular hole, foveal retinoschisis, idiopathic central serous chorioretinopathy, choroidal neovascular membrane, age-related macular degeneration and pigment epithelial detachment.

The use of contact lens fundoscopy and OCT macula scans helped complete the diagnosis of CME OS. The concurrent anterior uveitis $\mathrm{OS}$ and the previous history of complex ocular surgeries aided in that diagnosis. The patient was educated on the conditions and the importance of compliance to ocular medications, future visits and possible Kenalog ${ }^{\circledR}$ (triamcinolone acetonide) injection if the ocular medications did not work. The patient was placed on Pred Forte ${ }^{\circledR}$ (prednisolone acetate) $1 \%$ every hour OS, Acular ${ }^{\mathbb{B}}$ (diclofenac) 4 times a day OS, and homatropine twice a day OS. The patient was educated to return to clinic at anytime in the future if problems occurred and given an Amsler grid to monitor vision.

\section{Follow-up visit \# I}

The patient returned to clinic on July 25th, 2005, with stable, decreased vision OS. The patient had no discomfort OS. He reported compliance to the treatment regimen. Pertinent findings included best corrected VA of 20/50 OS. Stable central distortion was seen on the Amsler grid OS. Slit lamp findings were normal OD. Conjunctiva/sclera showed no injection OS. The cornea OS still had fine keratic precipitates corneal endothelium OS. Anterior chamber angle was deep with trace cells and flare OS. IOP was $15 \mathrm{mmHg}$ OD and OS with applanation tonometry. Dilated fundus exam was stable OU with the macula showing thickening with cystoid 


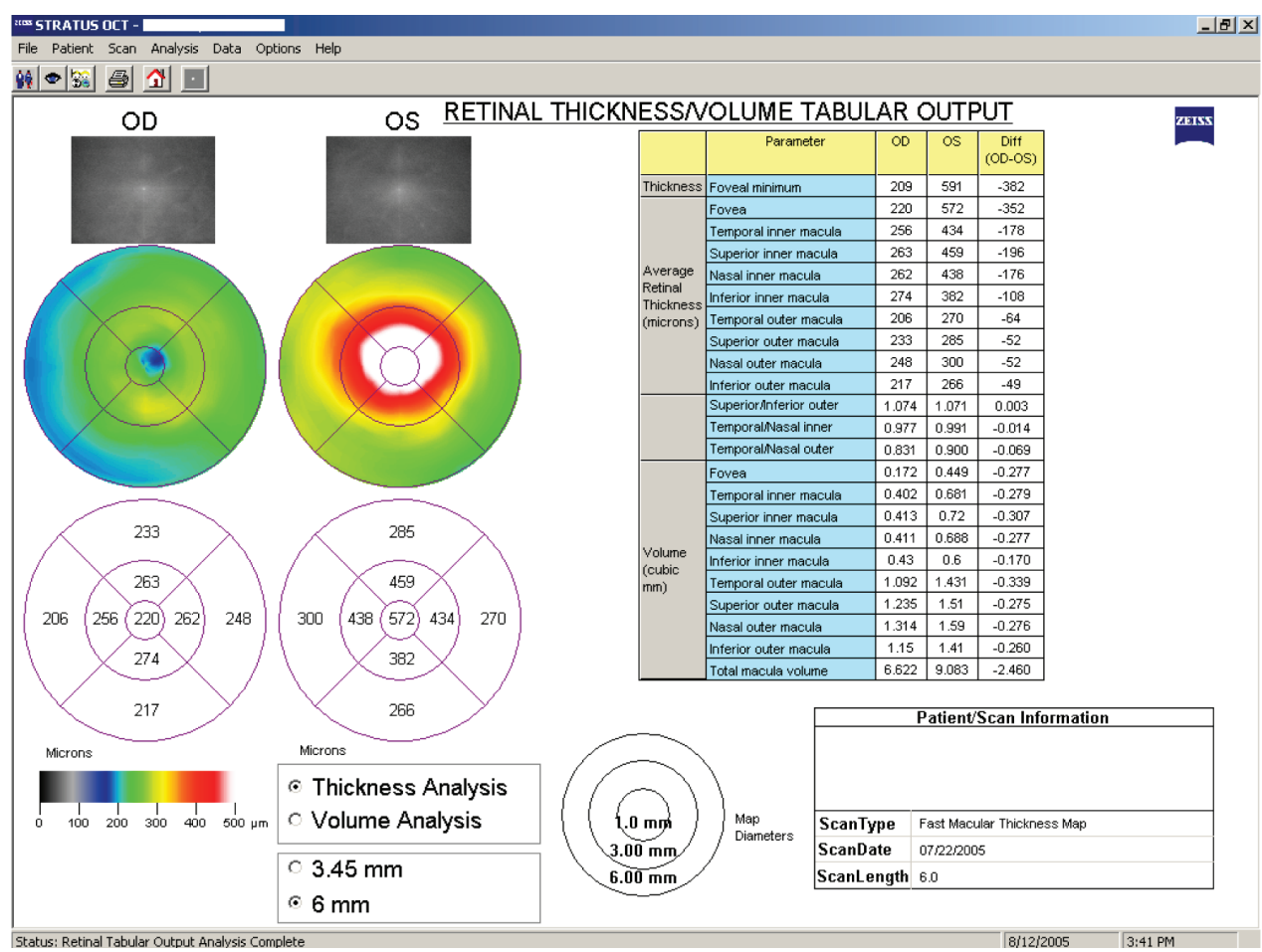

Figure 2 Screenshot of OCT Macula scan results from initial visit July 22, 2005.

Abbreviation: OCT, optical coherence tomography.

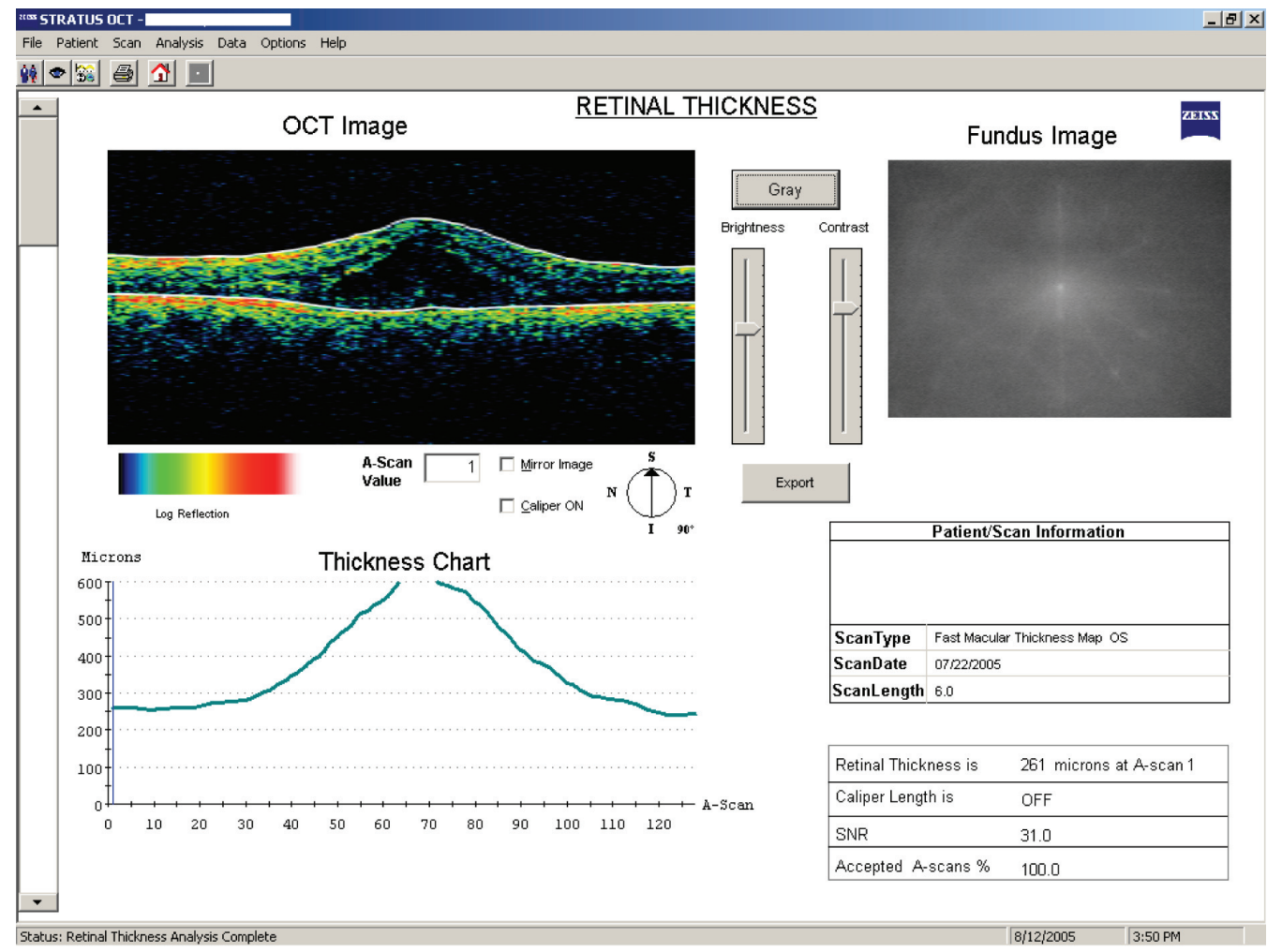

Figure 3 Screenshot of OCT Line scan OS from initial visit July 22, 2005.

Abbreviations: $\mathrm{OCT}$, optical coherence tomography; $\mathrm{OS}$, oculus sinister. 
spots OS. An OCT macula scan showed a foveal thickness of $520 \mu \mathrm{m}$ OS (Figure 4). The patient was again assessed with CME OS and improving anterior uveitis OS. The patient was to continue prednisolone acetate $1 \%$ every 2 hours OS, diclofenac 4 times a day OS, and homatropine twice a day OS. Due to the minimal effects the patient was to return to the clinic August 3rd, 2005, for intravenous FA and ophthalmology consult for possible triamcinolone acetonide injection if no improvement was seen with the CME OS.

\section{Follow-up visit \#2}

The patient returned to the clinic on August 3rd, 2005, with stable, decreased vision OS. The ophthalmologist did not perform FA due to elevated blood pressure and systemic hypertension after the patient was given $10 \%$ phenylephrine. Therefore, the patient was seen by the author instead. The patient again reported compliance to the treatment regimen. Pertinent findings included best corrected VA of 20/40 OS. Stable central distortion was seen on Amsler grid OS. Slit lamp findings were normal OD. Conjunctiva/sclera showed no injection OS. The cornea OS still had fine keratic precipitates corneal endothelium OS. Anterior chamber angles were deep and quiet OU. IOP was $15 \mathrm{mmHg}$ $\mathrm{OD}$ and $\mathrm{OS}$ with applanation tonometry. Dilated fundus exam was stable $\mathrm{OU}$ with the macula showing reduced thickening with cystoid spots OS. An OCT macula scan showed a foveal thickness of $367 \mu \mathrm{m}$ OS. The patient was assessed with improving CME OS and improving anterior uveitis OS. The options were discussed with the patient. The patient reported that he wanted to continue with the topical treatment and continue care with the author. The patient was to continue prednisolone acetate $1 \%$ 4 times a day OS, diclofenac 4 times a day OS, homatropine twice a day OS. The triamcinolone acetonide injection was not needed due to decreased swelling on OCT, improved ocular comfort, and improved VA. The patient was to return to the clinic on August 10th, 2005, for follow-up.

\section{Follow-up visit \#3}

The patient returned to the clinic on August 10th, 2005, with improved vision and Amsler grid results OS. The patient again reported compliance to the treatment regimen. Pertinent findings included best corrected VA of 20/25 OS. Decreased central distortion was seen on Amsler grid OS. Slit-lamp findings were normal OU. IOP was $15 \mathrm{mmHg}$ OD and OS with applanation tonometry. Dilated fundus exam was stable OU with the macula showing reduced thickening with cystoid spots OS. An OCT macula scan showed a foveal thickness of $280 \mu \mathrm{m}$ OS. The patient was again assessed with improving CME OS and resolved anterior uveitis OS.

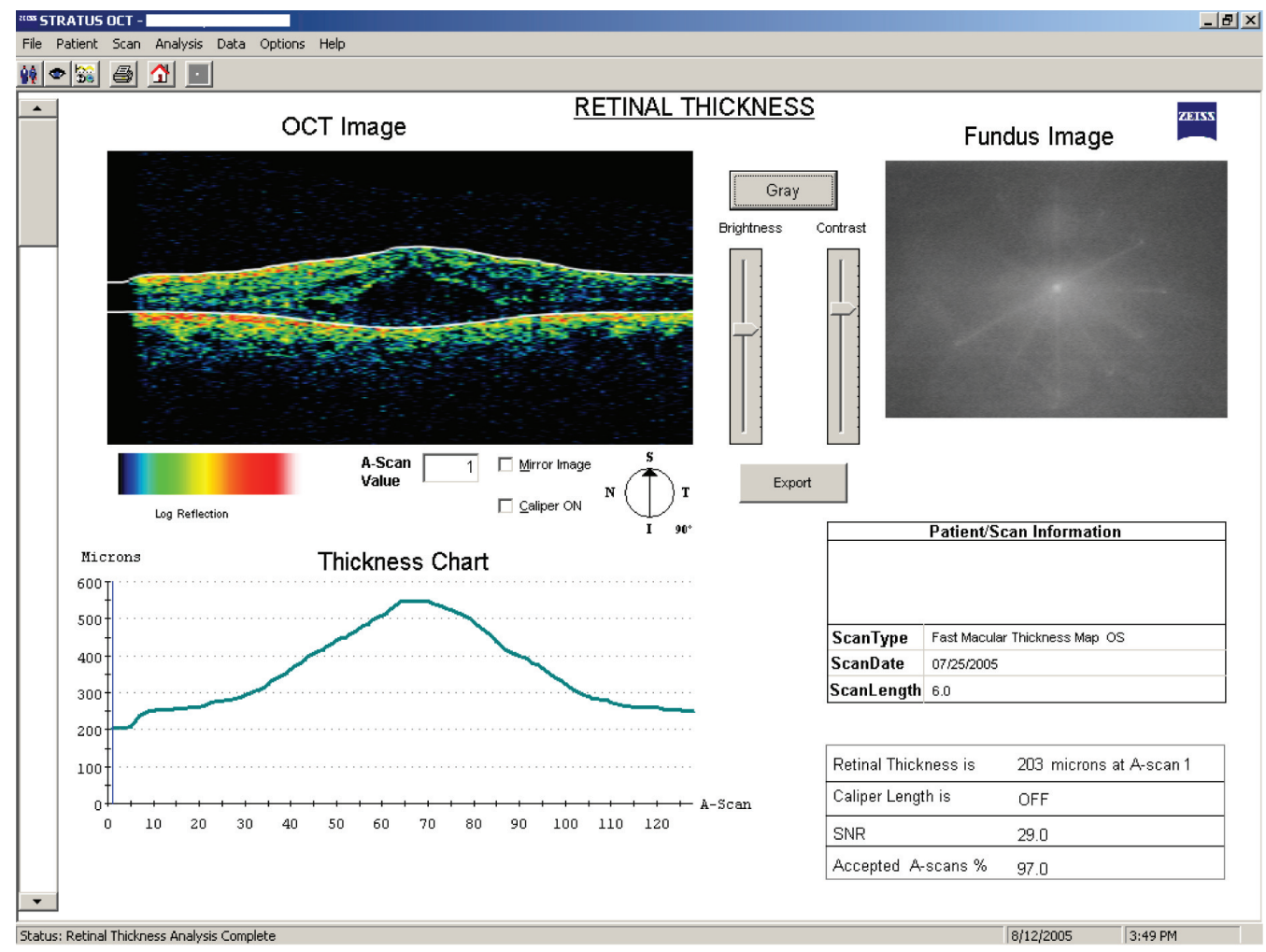

Figure 4 Screenshot of OCT Line scan OS follow-up visit \#I July 25, 2005. Abbreviations: OCT, optical coherence tomography; OS, oculus sinister. 


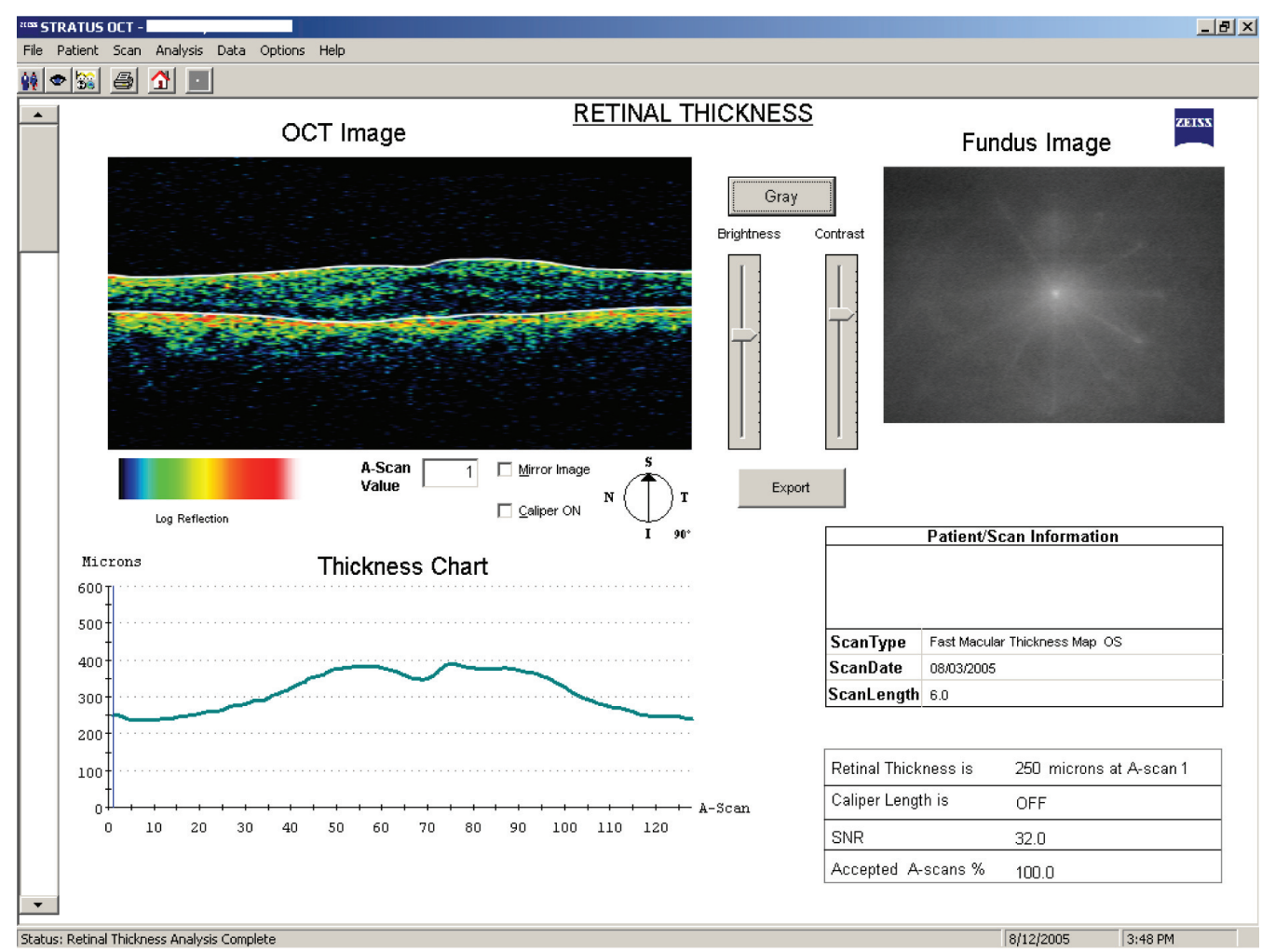

Figure 5 Screenshot of OCT Line scan OS follow-up visit \#2 August 3, 2005.

Abbreviations: OCT, optical coherence tomography; OS, oculus sinister.

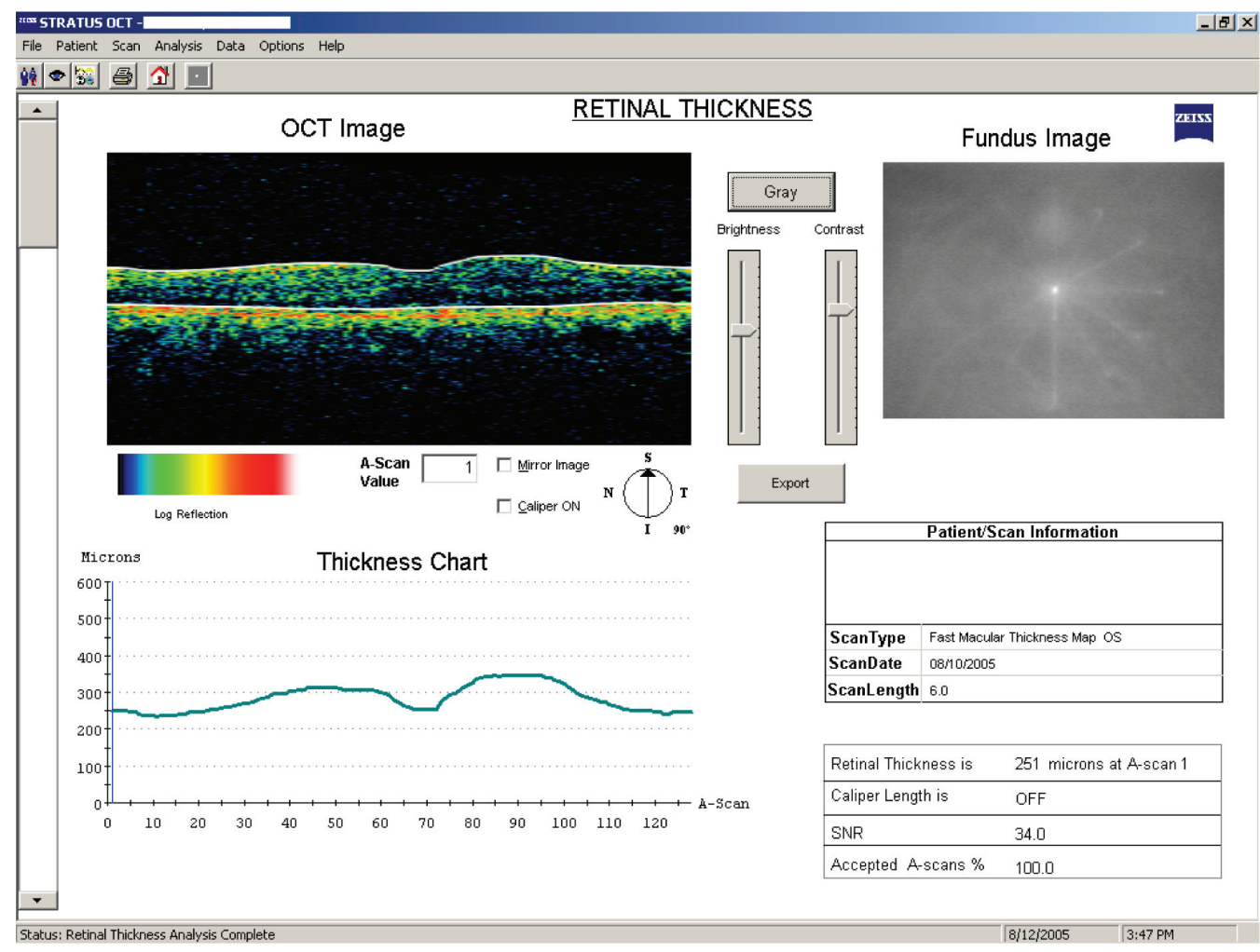

Figure 6 Screenshot of OCT Line scan OS follow-up visit \#3 August 10, 2005.

Abbreviations: OCT, optical coherence tomography; OS, oculus sinister. 


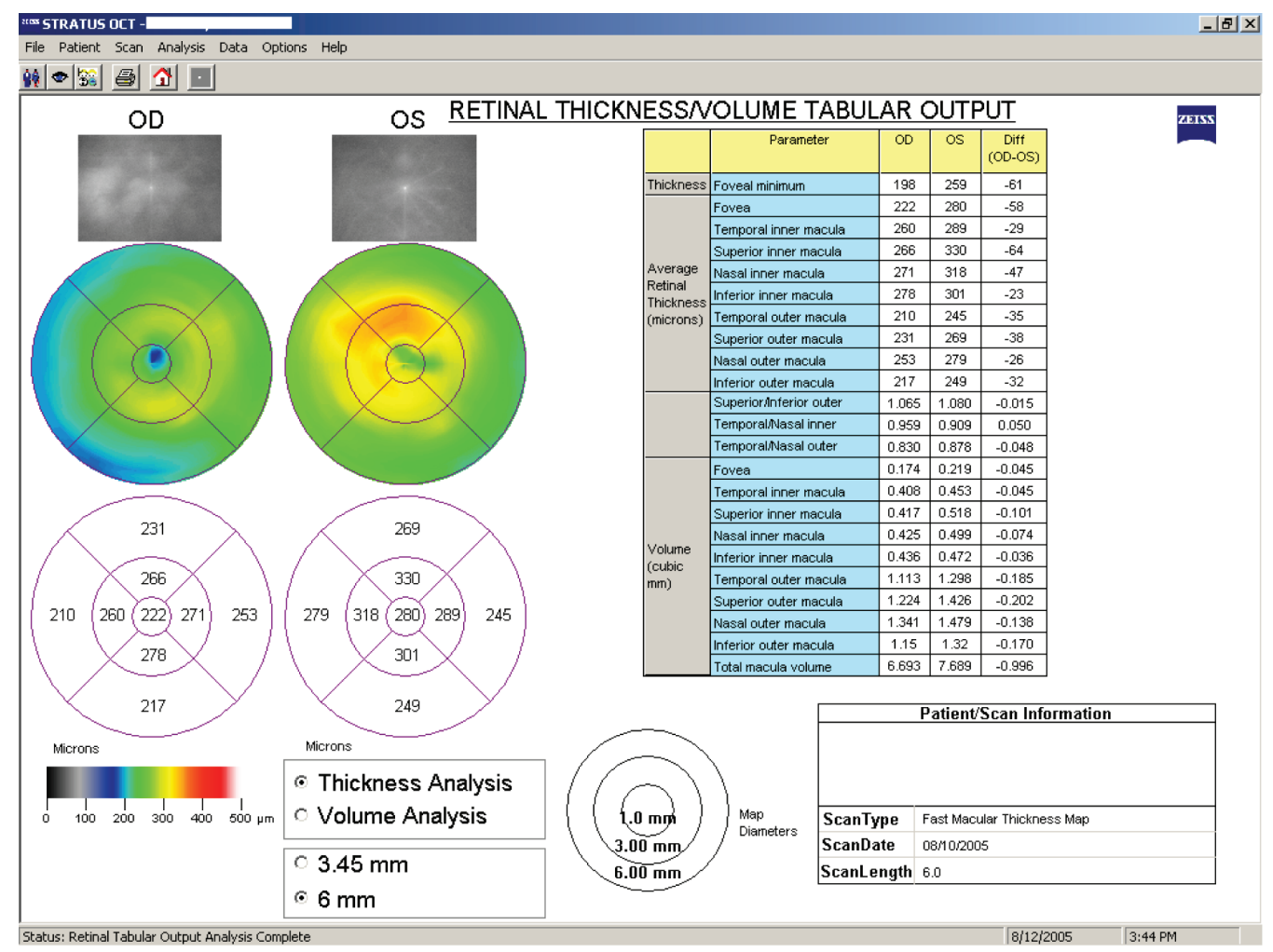

Figure 7 Screenshot of OCT macula scans showing foveal thickness at visit \#3 August 10, 2005. Abbreviations: OCT, optical coherence tomography; OS, oculus sinister; OD, oculus dexter.

The options were discussed with the patient. The patient reported that he wanted to continue with topical treatment and continue care with the author. The patient was to continue prednisolone acetate $1 \% 4$ times a day OS, diclofenac 4 times a day OS, homatropine twice a day OS. A triamcinolone acetonide injection not needed due to decreased swelling on OCT, improved ocular comfort, and improved VA. The patient was to return to the clinic on August 17th, 2005, for follow-up. Unfortunately the patient was then lost to follow-up.

\section{Discussion}

\section{Cystoid macular edema}

$\mathrm{CME}$ is characterized by inflammation at the fovea caused by the breakdown of the blood-retinal barrier, leading to the accumulation of fluid-filled cysts in the outer plexiform and inner nuclear layers of the retina. ${ }^{7}$ Physiologically, CME is caused by prostaglandin release, resulting in retinal vasodilation and vasopermeability.

The etiology of CME is most often due to a history of any type of ocular surgery, especially any form of intraocular or complex ocular surgery no matter how long ago. CME is most often seen postcataract surgery (even uncomplicated surgeries) and is referred to as Irvine-Gass syndrome in that situation. Peak incidence of CME after cataract surgery occurs about 6 weeks after postoperatively. The probability of CME after cataract surgery increases when the posterior capsule is ruptured or if there is vitreous prolapse. Other common causes of CME include diabetic retinopathy, retinal vein occlusions, and uveitis. ${ }^{1-3}$ Less common causes include drug induced (such as topical phenylephrine or prostaglandin use), ${ }^{8}$ retinal dystrophies such as retinitis pigmentosa, and vitreomacular traction. In this case presentation, the patient had a history of several complex intraocular surgeries and had a subsequent anterior uveitis inflammation which helped in the diagnosis of CME.

The clinical presentation of CME includes a loss of the foveal light reflex, macular thickening and microcysts. This patient presented with all of those characteristics. The use of contact-lens fundoscopy and OCT proved useful in the initial examination of this patient. Contact-lens fundoscopy allowed this examiner to directly view the elevation and the layers of microcysts at the fovea.

In retrospect, a FA was desired although not needed due to other parts of the examination and the patient's elevated blood pressure. Historically, FA has been the main diagnostic test for CME. The accumulation of fluorescein dye in the 
outer plexiform layer of the retina, with its radial arrangement of fibers around the fovea, led to the distinguishing flowerpetal pattern.

\section{Advantages of OCT in the diagnosis and management of CME (and other macular diseases)}

OCT has revolutionized standard ophthalmic care as it uses high resolution cross-sectional imaging to provide a quantitative measure of retinal thickness. Compared to intravenous FA, OCT is noninvasive and quantitative (nonsubjective). ${ }^{9}$ Before the use of OCT in clinical practice, only qualitative methods were used to detect changes in macular thickness such as stereoscopic photography and slit lamp biomicroscopy. OCT now provides a quantitative method for analyzing retinal thickness, which in this and many other cases provides for more accurate management of conditions with changes in retinal thickness.

The OCT fast macular thickness function is the most common way to measure retinal thickness within the macular area. It uses cross-sectional scans consisting of six radial lines, each passing through the foveal center. ${ }^{10}$ The total OCT macular map covers a $6 \mathrm{~mm}$ diameter circle around the foveal center. The scan time of the OCT fast macular thickness scan takes about 1.92 seconds per scan and performs 128 A-scans per optical cross section. ${ }^{10}$ The OCT has been shown to be accurate to within 10-14 $\mu \mathrm{m} .{ }^{5,11,10}$ Also, OCT has been reported to be $89 \%-96 \%$ sensitive in the detection of CME and 100\% specific. In this patient's case, OCT proved to be an invaluable tool for the diagnosis and management of CME especially when FA could not be performed due to systemic hypertension and the elevated blood pressure from 10\% phenylephrine.

\section{Topical treatment of CME}

This patient presented with a mild anterior uveitis along with the CME OS. With uveitic inflammation observed, it was determined to utilize the topical treatments listed below, to not only treat the uveitis, but also to see if the CME could be managed topically.

Nonsteroidal anti-inflammatory drugs (NSAIDs) are the first line of therapy ${ }^{12-14}$ that has approval and are used for the treatment and prevention of CME after ocular surgeries. One example of an ophthalmic NSAID is diclofenac which inhibits prostaglandin formation through cyclooxygenase-1 and cyclooxygenase- $2 .{ }^{12}$

Due to the concurrent anterior uveitis in this case, $1 \%$ prednisolone acetate was also used to treat not only the anterior uveitis but also to see if it could help with the retinal cystoid edema. Prednisolone acetate is a corticosteroid. Corticosteroids inhibit cyclooxygenases and interleukin-1 in the inflammatory cascade to help decrease vascular permeability. The use of prednisolone acetate has been shown to be very effective when used adjacent to NSAIDs. ${ }^{12,14}$

\section{Other possible treatments for CME}

Some other possible treatments for $\mathrm{CME}$ included the subtenon injection of triamcinolone acetonide, ${ }^{15,16}$ oral and IV steroids, oral NSAIDs.

Oral NSAIDs can be considered for treatment if no response is seen with topical treatments. Indomethacin ( $25 \mathrm{mg}$ by mouth per day for 6-8 weeks) is the treatment of choice for oral NSAIDs. If oral steroids are to be used, it should be prednisone $40-60 \mathrm{mg}$ by mouth 4 times per day for 2 weeks, then followed by a slow taper. Also, oral acetazolomide (250 $\mathrm{mg}$ by mouth twice a day) has been used. ${ }^{17}$ However, these oral treatments are all somewhat unproven. ${ }^{17,18}$

With this patient, a triamcinolone acetonide injection was not needed due to the decreased CME measured with OCT, improved ocular comfort, and improved VA with diclofenac and prednisolone acetate drops.

\section{Conclusion}

This case provides a review of CME and indications for ocular treatment. Also, OCT macular scans were used in this case to not only diagnose CME but also to better evaluate the treatment and management of this case. Now and in the future, OCT will provide more accurate analysis for the diagnosis and management of $\mathrm{CME}$ and other macular disorders.

\section{Disclosure}

The author reports no conflicts of interest relevant to this study.

\section{References}

1. Reinthal EK, Volker M, Freudenthaler N, et al. Optical coherence tomography in the diagnosis and follow-up of patients with uveitic macular edema. Ophthalmologe. 2004;101(12):1181-1188.

2. Estafanous MF, Lowder CY, Kaiser PK. Patterns of macular edema in uveitis patients. Ophthalmology. 2005;112(2):360. Author reply -1 .

3. Quinn CJ. Cystoid macular edema. Optom Clin. 1996;5(1):111-130.

4. Guex-Crosier Y. The pathogenesis and clinical presentation of macular edema in inflammatory diseases. Doc Ophthalmol. 1999;97(3-4): 297-309.

5. Schaudig U, Scholz F, Lerche RC, Richard G. Optical coherence tomography for macular edema. Classification, quantitative assessment, and rational usage in the clinical practice. Ophthalmologe. 2004; 101(8):785-793.

6. Bouma BE, Tearney GJ. Handbook of Optical Coherence Tomography. New York, NY: Marcel Dekker, 2002; p. 741. 
7. Sebag J, Balazs EA. Pathogenesis of cystoid macular edema: an anatomic consideration of vitreoretinal adhesions. Surv Ophthalmol. 1984;28 Suppl:493-498.

8. Warwar RE, Bullock JD, Ballal D. Cystoid macular edema and anterior uveitis associated with latanoprost use. Experience and incidence in a retrospective review of 94 patients. Ophthalmology. 1998;105(2): 263-268.

9. Thomas D, Duguid G. Optical coherence tomography - a review of the principles and contemporary uses in retinal investigation. Eye 2004; 18(6):561-570.

10. Polito A, Del Borrello M, Isola M, et al. Repeatability and reproducibility of fast macular thickness mapping with stratus optical coherence tomography. Arch Ophthalmol. 2005;123(10):1330-1337.

11. Ching HY, Wong AC, Wong CC, et al. Cystoid macular oedema and changes in retinal thickness after phacoemulsification with optical coherence tomography. Eye. 2006;20(3):297-303.

12. Singal N, Hopkins J. Pseudophakic cystoid macular edema: ketorolac alone versus ketorolac plus prednisolone. Can J Ophthalmol. 2004; 39(3):245-250.
13. Sivaprasad S, Bunce C, Wormald R. Non-steroidal anti-inflammatory agents for cystoid macular oedema following cataract surgery: a systematic review. Br J Ophthalmol. 2005;89(11):1420-1422.

14. Thurau SR. Cystoid macular edema in uveitis. Ophthalmologe. 2005; 102(5):485-490.

15. Angunawela RI, Heatley CJ, Williamson TH, et al. Intravitreal triamcinalone acetonide for refractory uveitic cystoid macular oedema: longterm management and outcome. Acta Ophthalmol Scand. 2005;83(5): 595-599.

16. Antcliff RJ, Spalton DJ, Stanford MR, et al. Intravitreal triamcinolone for uveitic cystoid macular edema: an optical coherence tomography study. Ophthalmology. 2001;108(4):765-772.

17. Pinckers A, Cruysberg JR, Kremer H, Aandekerk AL. Acetazolamide in dominant cystoid macular dystrophy. A pilot study. Ophthalmic Paediatr Genet. 1993;14(2):95-99.

18. Kaiser PK, Friedman NJ, Pineda R, Massachusetts Eye and Ear Infirmary Illustrated Manual of Ophthalmology, 2nd ed. Philadelphia, PA: Saunders; 2004. p. 537.
Clinical Optometry

\section{Publish your work in this journal}

Clinical Optometry is an international, peer-reviewed, open access journal publishing original research, basic science, clinical and epidemiological studies, reviews and evaluations on clinical optometry. All aspects of patient care are addressed within the journal as well as the practice of optometry including economic and business analyses. Basic and clinical

\section{Dovepress}

research papers are published that cover all aspects of optics, refraction and its application to the theory and practice of optometry. The manuscript management system is completely online and includes a very quick and fair peer-review system, which is all easy to use. Visit http://www.dovepress. com/testimonials.php to read real quotes from published authors. 